Mathematical Research Letters 4, 201-210 (1997)

\title{
DISKBUSTING ELEMENTS OF THE FREE GROUP
}

\author{
RichaRD STONG
}

\begin{abstract}
In 1936 Whitehead presented an algorithm for determining whether an element (or a set of elements) in a free group $F$ is part of a free generating set for $F$. We will give a more detailed discussion of Whitehead's algorithm and a new proof that the algorithm works. We will see that in fact Whitehead's algorithm actually determines whether or not an element (or a set of elements) is diskbusting. If an element $\omega$ is not diskbusting, then Whitehead's algorithm produces the smallest free factor of $F$ in which $\omega$ lies, and in that free factor $\omega$ is diskbusting.
\end{abstract}

\section{Introduction}

Let $F_{n}$ be the free group on $n$ generators $a_{1}, a_{2}, \ldots, a_{n}$ and their inverses $a_{1}^{\prime}, a_{2}^{\prime}, \ldots, a_{n}^{\prime}$. We will call $a_{1}, a_{2}, \ldots, a_{n}, a_{1}^{\prime}, a_{2}^{\prime}, \ldots, a_{n}^{\prime}$ the letters of $F_{n}$. If $x$ is a letter of $F_{n}$, then we will denote its inverse by $x^{\prime}$ and hence $x^{\prime \prime}=x$. An element of $F_{n}$ is a word in the letters of $F_{n}$ and a conjugacy class in $F_{n}$ may be regarded as being a cyclically ordered word in the letters of $F_{n}$. We will abuse terminology slightly and refer to a cyclic word in the letters of $F_{n}$ as a cyclic word in $F_{n}$. We will say a word or cyclic word is reduced if it contains no occurrence of $x x^{\prime}$ for $x$ a letter. Recall that any word or cyclic word can be cancelled down to a unique reduced word.

Suppose $\omega=z_{1} z_{2} \ldots z_{r}$ is a reduced cyclic word in the letters of $F_{n}$. Since $\omega$ is cyclically ordered, we can define $z_{i}$ for any integer $i$ by the rule $z_{i+r}=z_{i}$. We will say $\omega$ is diskbusting if there is no free product decomposition $F_{n}=A * B$ with $B$ nontrivial and $\omega$ conjugate into $A$. This has a geometric interpretation as well. Represent $F_{n}$ as the fundamental group of a 3-dimensional handlebody $H$. Then $\omega$ determines a free homotopy class of closed loops in $H$. Then $\omega$ is diskbusting if for any essential 2-disk $D$ in $H$ and any closed loop $\gamma$ in $H$ representing $\omega, \gamma$ meets $D$. Diskbusting curves play an important role in the study of 3 -manifolds, e.g., [1]. More generally, if $\Omega=\left\{\omega_{1}, \omega_{2}, \ldots\right\}$ is a finite set of cyclic words, we say $\Omega$ is diskbusting if there is no non-trivial free product decomposition $F_{n}=A * B$ with each $\omega_{i}$ conjugate into either $A$ or $B$. (Here a decomposition is trivial if one of $A$ and $B$ is trivial and every $\omega_{i}$ is conjugate into the other.) Again this

Received October 11, 1996.

The author was partially supported by an NSF Postdoctoral Fellowship and an Alfred P. Sloan Fellowship. 
has a similar geometric interpretation. For any representatives $\gamma_{i}$ of $\omega_{i}$ and any essential 2-disk $D$ at least one of the $\gamma_{i}$ must meet $D$.

An obvious question one can ask is whether a given finite set $\Omega$ of cyclic words is diskbusting. We will show that the answer to this question is decidable by an easy algorithm. In fact, except for the very end, our algorithm is identical to the algorithm given by Whitehead in 1936 to decide whether $\Omega$ is part of a free basis. This paper can be regarded as giving a more detailed study of Whitehead's algorithm and as a bonus we will give a new proof of Whitehead's algorithm.

The questions of whether a finite set $\Omega=\left\{\omega_{1}, \omega_{2}, \ldots\right\}$ is diskbusting or part of a free basis can also be asked if the $\omega_{i}$ are ordinary, i.e., not cyclic, words. There are two distinct notions of diskbusting one can use in this case. Algebraically, we can say $\Omega$ is (algebraically) diskbusting if there is no non-trivial decomposition $F_{n}=A * B$ with each $\omega_{i}$ in either $A$ or $B$. (Here a decomposition is trivial if one of $A$ and $B$ is trivial and every $\omega_{i}$ is in the other.) Geometrically, we can fix a base point $\star$ in $H$ and represent each $\omega_{i}$ by a based loop $\gamma_{i}$. We can say $\Omega$ is (geometrically) diskbusting if for any choice of $\gamma_{i}$ and any essential 2-disk $D \subset H, D$ meets at least one of the $\gamma_{i}$. These two notions of diskbusting do not agree. The algebraic interpretation of "geometrically diskbusting" is that there is no decomposition $F_{n}=A * B$ with each $\omega_{i}$ in $A$ and $B \neq\{1\}$. We sketch the extension to this case in the last section.

\section{Cyclic words}

If $\omega=z_{1} z_{2} \ldots z_{r}$ is a cyclic word we can associate to $\omega$ a family of biinfinite paths in $F_{n},\left\{\ldots z_{2}^{-1} z_{1}^{-1} g, z_{1}^{-1} g, g, z_{r} g, z_{r-1} z_{r} g, \ldots\right\}$. We will call these paths $\omega-$ geodesics. The geometric interpretation of these $\omega$-geodesics is as follows. Fix a hyperbolic structure on $H$ and choose $n$ totally geodesic 2-disks $D_{1}^{2}, D_{2}^{2}, \ldots, D_{n}^{2}$ dual to $a_{1}, a_{2}, \ldots, a_{n}$, so that the element of $F_{n}$ represented by a loop in $H$ can be read off by recording $a_{i}$ (resp. $a_{i}^{-1}$ ) if the loop crosses the 2-disk $D_{i}^{2}$ positively, (resp. negatively). Cutting along the $D_{i}^{2}$ produces a unit cell $X$ and the universal cover $\tilde{H}$ is built by gluing up countably many copies of $X$ indexed by $F_{n}$. Take any lift $\tilde{\gamma}$ of a geodesic representing $\omega$. Then the record of the copies of $X$ which $\tilde{\gamma}$ passes through is exactly an $\omega$-geodesic in $F_{n}$. If $\omega$ has extra cyclic symmetry, then these geodesics are repeated. In this case we will count them with the appropriate multiplicity. Thus for any element $h$ of $F_{n}$ there are $r \omega$-geodesics which go through $h$, namely $\left\{\ldots z_{i+2}^{-1} z_{i+1}^{-1} h, z_{i+1}^{-1} h, h, z_{i} h, z_{i-1} z_{i} h, \ldots\right\}, 1 \leq i \leq r$.

We can use the $\omega$-geodesics to associate a family of graphs to the element $\omega$. There is a natural metric on $F_{n}$, the word metric determined by the letters $a_{1}, a_{2}, \ldots, a_{n}, a_{1}^{\prime}, a_{2}^{\prime}, \ldots, a_{n}^{\prime}$. Let $S_{R}$ be the sphere of radius $R$ about the identity element in this metric and $B_{R}$ the ball of radius $R$. Let $\Gamma_{R}(\omega)$ be the graph whose vertex set is $S_{R}$ and where $g_{1}, g_{2} \in S_{R}$ are joined by one edge for each $\omega$-geodesic on which they both lie. Geometrically this corresponds to taking the compact piece $K$ of the universal cover $\tilde{H}$ which consists of the union of the copies of $X$ corresponding to $B_{R-1}$. For the vertices we collapse the boundary 
2-disks of $K$, which correspond to $S_{R}$, to points. For the edges we take all the lifts of a geodesic representing $\omega$ in $K$. If $\Omega=\left\{\omega_{1}, \omega_{2}, \ldots\right\}$ is a finite set of cyclic words, then we let $\Gamma_{R}(\Omega)$ have the same vertex set and its edge set is the disjoint union of the edge sets of the $\Gamma_{R}\left(\omega_{i}\right)$.

The graph $\Gamma_{1}(\Omega)$ is the Whitehead graph of $\Omega$ introduced by Whitehead in [2]. There is actually a slight difference, Whitehead introduced extra vertices to subdivide each edge of $\Gamma_{1}(\Omega)$. This change is used by Whitehead to get a more homogeneous statement. With our new interpretation we will not need it. The Whitehead graph can be described as having for its vertices the letters of $F_{n}$ and for each pair $x y$ of consecutive letters in some $\omega_{i}$ we have an edge from $x$ to $y^{\prime}$. Note that the $r$ edges from $\omega$ given by this rule exactly correspond to the $r$ $\omega$-geodesics through 1 described above.

There are several useful properties of the graphs $\Gamma_{R}(\Omega)$. Note that we have a projection map $p: \Gamma_{R}(\Omega) \rightarrow \Gamma_{R-1}(\Omega)$ given by sending $x_{1} x_{2} \ldots x_{R}$ to $x_{2} x_{3} \ldots x_{R}$. An edge in $\Gamma_{R-1}(\Omega)$ joining $x_{2} x_{3} \ldots x_{R}$ to $y_{2} y_{3} \ldots y_{R}$ corresponds to a segment in an $\omega_{i}$-geodesic. The next two vertices out on that $\omega_{i}$-geodesic are of the form $x_{1} x_{2} \ldots x_{R}$ and $y_{1} y_{2} \ldots y_{R}$. Thus they are vertices of $\Gamma_{R}(\Omega)$ joined by an edge. Thus the projection $p$ is surjective on the edges as well as on the vertices. Also note that for every $x_{2} x_{3} \ldots x_{R}$ the subgraph $p^{-1}\left(x_{2} x_{3} \ldots x_{R}\right)$ has vertices $\left\{t x_{2} x_{3} \ldots x_{R}: t \neq x_{2}^{-1}\right\}$ and $\Gamma_{R}(\Omega)$ restricted to these vertices is a copy of $\Gamma_{1}(\Omega)-\left\{x_{2}^{-1}\right\}$. Thus we can describe $\Gamma_{R-1}(\Omega)$ as being obtained from $\Gamma_{R}(\Omega)$ by collapsing a large number of copies of $\Gamma_{1}(\Omega)-\{x\}$, for different $x$, to vertices.

More generally let $T_{n}$ be the infinite $2 n$-regular tree which is the Cayley graph of $F_{n}$ with respect to the generating set $\left\{a_{1}, a_{2}, \ldots, a_{n}, a_{1}^{\prime}, a_{2}^{\prime}, \ldots, a_{n}^{\prime}\right\}$. For any compact subset $K \subset T_{n}$ we can form a graph $\Gamma_{K}(\Omega)$ as follows. The vertices of $\Gamma_{K}(\Omega)$ are the unbounded components of $T_{n}-K$ and for each $\omega_{i^{-}}$ geodesic $\gamma$ we have an edge joining the unbounded component of $T_{n}-K$ where $\gamma$ begins to the one where $\gamma$ ends. Note that this definition is in some sense more natural since we do not need to assume $\omega_{i}$ is reduced. This definition generalizes immediately to compact subsets $K \subset \tilde{H}$. Also this extends the previous definition since $\Gamma_{R}(\Omega)=\Gamma_{B_{r-1}}(\Omega)$. Furthermore if $K^{\prime} \subset K$ then we have a projection $\Gamma_{K}(\Omega) \rightarrow \Gamma_{K^{\prime}}(\Omega)$ which is surjective on vertices and edges.

In some weak sense this definition shows the family of $\Gamma_{R}(\Omega)$ are independent of the particular generating set for $F_{n}$. Let $b_{1}, b_{2}, \ldots, b_{n}$ be another free basis for $F_{n}, \hat{S}_{R}$ and $\hat{B}_{R}$ the spheres and balls, $\hat{T}_{n}$ the tree, and let $\hat{\Gamma}_{R}(\Omega)$ be the graphs associated to that basis. There is a constant $C>0$ such that any generator $a_{j}$ has length at most $C$ when written in terms of $\left\{b_{i}\right\}_{i=1}^{n}$ and conversely. Then any element of $F_{n}-B_{C(R+C)}$ is in $F_{n}-\hat{B}_{R+C}$. Thus any unbounded component of $T_{n}-B_{C(R+C)}$ lies in a single unbounded component of $\hat{T}_{n}-\hat{B}_{R}$. Hence we have projections $\Gamma_{C(R+C)}(\Omega) \rightarrow \hat{\Gamma}_{R}(\Omega)$ and $\hat{\Gamma}_{C(R+C)}(\Omega) \rightarrow \Gamma_{R}(\Omega)$. In particular if $\Gamma_{R}(\Omega)$ is connected for arbitrarily large $R$, then so is $\hat{\Gamma}_{R}(\Omega)$. (Alternately there is a basis independent inverse limit of the graphs $\Gamma_{R}(\Omega)$. Although all results can be phrased in terms of this infinite object we will work with the infinite 
families of finite objects instead.)

For a graph $\Gamma$ and a vertex $x$ of $\Gamma$ we will denote by $\operatorname{deg}(x)$ the number of edges of $\Gamma$ incident on $x$. We will say a graph $\Gamma$ is 1 -connected if $\Gamma$ is connected and remains connected even if any one vertex is removed. If $\Gamma$ is connected and not 1-connected, then we call any vertex whose removal disconnects $\Gamma$ a cut vertex. Isolated vertices in $\Gamma_{1}(\Omega)$ correspond to letters not used by any $\omega_{i}$ and occur in letter/inverse pairs. Whitehead [2] assumes that such isolated vertices of $\Gamma_{1}(\Omega)$ are removed. We do not make this assumption. With this slight change in terminology we have the following version of Whitehead's Reduction Lemma $[2]$.

Lemma 1 (Whitehead). If $\Gamma_{1}(\Omega)$ has a vertex $x$ and set of fewer than deg $(x)$ edges whose removal disconnects $\Gamma_{1}(\Omega)$ and separates $x$ from $x^{\prime}$, then we may change bases of $F_{n}$ and shorten $\Omega$, i.e., decrease the sum of the lengths of the $\omega_{i}$.

Proof. Suppose $\operatorname{deg}(x)>k$ and there are $k$ edges $\left\{e_{1}, e_{2}, \ldots, e_{k}\right\}$ which separate $x$ from $x^{\prime}$. Let $\left\{x, y_{1}, y_{2}, \ldots, y_{r}\right\}$ be the component of $\Gamma_{1}(\omega)-\left\{e_{1}, e_{2}, \ldots, e_{k}\right\}$ containing $x$, but not $x^{\prime}$. We may suppose that no $e_{i}$ joins two vertices in $\left\{x, y_{1}, y_{2}, \ldots, y_{r}\right\}$, that $\left\{e_{1}, e_{2}, \ldots, e_{l}\right\}$ do not have $x$ as a vertex and that $\left\{e_{l+1}, e_{l+2}, \ldots, e_{k}\right\}$ have $x$ as a vertex. Suppose $x=a_{i}^{\epsilon}$. Make the following change of basis $\tilde{a}_{i}=a_{i}$ and if $j \neq i$,

$$
\tilde{a}_{j}= \begin{cases}x a_{j} x^{\prime}, & \text { if } a_{j} \text { and } a_{j}^{\prime} \in\left\{y_{1}, y_{2}, \ldots, y_{r}\right\}, \\ a_{j} x^{\prime}, & \text { if only } a_{j} \in\left\{y_{1}, y_{2}, \ldots, y_{r}\right\}, \\ x a_{j}, & \text { if only } a_{j}^{\prime} \in\left\{y_{1}, y_{2}, \ldots, y_{r}\right\}, \\ a_{j}, & \text { if } a_{j} \text { and } a_{j}^{\prime} \notin\left\{y_{1}, y_{2}, \ldots, y_{r}\right\} .\end{cases}
$$

When we rewrite $\Omega$ in this new basis, we add an $x$ after every occurrence of $y_{i}$, add an $x^{\prime}$ before every occurrence of $y_{i}^{\prime}$, put tildes over all the letters, and reduce. Except for $l$ places in $\Omega$ (corresponding to $\left\{e_{1}, e_{2}, \ldots, e_{l}\right\}$ ), any occurrence of $y_{i}$ is followed by an element of $\left\{x^{\prime}, y_{1}^{\prime}, \ldots, y_{k}^{\prime}\right\}$ and any occurrence of $y_{i}^{\prime}$ is preceded by an element of $\left\{x, y_{1}, \ldots, y_{k}\right\}$. Therefore all but at most $l$ of the new $x$ or $x^{\prime}$ added are immediately cancelled in the reduction step. Further each occurrence of $x y_{i}^{\prime}$ or $y_{i} x^{\prime}$ in $\Omega$ leads to a shortening of $\Omega$ when we reduce. There are $\operatorname{deg}(x)-(k-l)$ such occurrences. Therefore the length of $\Omega$ decreases by at least $\operatorname{deg}(x)-k$ (though there may be more cancellation). At any rate, we have succeeded in shortening $\Omega$.

As a consequence of this Lemma we may shorten $\Omega$ if $\Gamma_{1}(\Omega)$ has a non-1connected component. (Take $x$ to be a cut vertex and $\left\{e_{1}, \ldots, e_{k}\right\}$ to be the edges joining $x$ to the component of $\Gamma_{1}(\Omega)-\{x\}$ containing $x^{\prime}$.) Alternately if $\Gamma_{1}(\Omega)$ has two components which both contain edges coming from the word $\omega_{i}$, then we may shorten $\Omega$. (Since both components contain edges coming from the same cyclic word $\omega_{i}$ there must be a generator $x$ in one component with $x^{\prime}$ in 
the other. Use this $x$ and $k=0$.) Applying this lemma and these two remarks repeatedly we get the following corollary.

Corollary 2. For any finite set $\Omega=\left\{\omega_{1}, \omega_{2}, \ldots\right\}$ of reduced cyclic words in $F_{n}$ of total length $L, \Omega$ can be shortened in at most $L-1$ iterations of the above reduction algorithm until $\Gamma_{1}(\Omega)$ consists of 1-connected components. Further, we may assume the edges coming from any $\omega_{i}$ lie in a single component.

Thus Whitehead's reduction lemma gives an algorithm for reducing $\Omega$ until it has a special form. We need to understand what we can learn from this special form. For example, Whitehead shows that if $\Omega$ is part of a basis for $F_{n}$, then the 1-connected components of $\Gamma_{1}(\Omega)$ must all be just a single edge. However there is more data available in this special form. Towards this end we have the following Theorem.

Theorem 3. Let $\Omega=\left\{\omega_{1}, \omega_{2}, \ldots\right\}$ be a finite set of reduced cyclic words in $F_{n}$. Then the following are equivalent.

(1) $\Gamma_{1}(\Omega)$ is 1-connected with respect to some basis for $F_{n}$.

(2) $\Gamma_{R}(\Omega)$ is connected for all $R$.

(3) $\Omega$ is diskbusting.

Condition (3) is clearly independent of the basis for $F_{n}$. Condition (2) is independent of the basis for $F_{n}$ by the discussion above. Condition (1) is independent of the basis only because that fact is explicitly inserted. It is possible for $\Gamma_{1}(\Omega)$ to be non-1-connected in one basis, but 1-connected in another. A specific example (with $\Omega$ a single word) is

$$
\omega=a^{2} b^{-1} a b^{-1} a^{-1} b a^{-1} c b^{-1} a c^{-1} a b^{-1} a^{-1} c a^{-1} b c^{-1} b c^{-1}
$$

which has $\Gamma_{1}(\omega)$ non-1-connected, but in the basis $\tilde{a}=a, \tilde{b}=b a^{-1}, \tilde{c}=c a^{-1}$ becomes

$$
\omega=\tilde{a} \tilde{b}^{-2} \tilde{a}^{-1} \tilde{b} \tilde{c} \tilde{b}^{-1} \tilde{c}^{-1} \tilde{b}^{-1} \tilde{a}^{-1} \tilde{c} \tilde{b} \tilde{c}^{-1} \tilde{b} \tilde{c}^{-1}
$$

which has $\Gamma_{1}(\Omega)$ 1-connected.

Proof. $(1) \Rightarrow(2)$. Fix a basis for $F_{n}$ in which $\Gamma_{1}(\Omega)$ is 1-connected. We will show (2) by induction on $R$. Suppose that contrary to the inductive step, $\Gamma_{R-1}(\Omega)$ is connected, but $\Gamma_{R}(\Omega)$ is not connected. In projecting from $\Gamma_{R}(\Omega)$ to $\Gamma_{R-1}(\Omega)$ we collapse a number of subgraphs $\Gamma_{1}(\Omega)-\{x\}$ for various choice of $x$ to points. Since all the $\Gamma_{1}(\Omega)-\{x\}$ are connected, these collapses cannot reconnect $\Gamma_{R}(\Omega)$. This is a contradiction.

$(2) \Rightarrow(3)$. Suppose $\Omega$ is not diskbusting. Then either there is a nontrivial free product decomposition $F_{n}=A * B$ with each $\omega_{i}$ conjugate into $A$ or $B$. In this basis $\Gamma_{R}(\Omega)$ is clearly disconnected for all $R$.

$(3) \Rightarrow(1)$. Suppose $\Gamma_{1}(\Omega)$ is never 1 -connected. Then by Lemma 3 we can find a basis in which $\Gamma_{1}(\Omega)$ consists of 1 -connected components (more then one) 
and each $\omega_{i}$ has edges in only a single component. This gives a nontrivial free product decomposition $F_{n}=A * B$ with each $\omega_{i}$ conjugate into $A$ or $B$.

This theorem gives a simple algorithm for determining whether an element or set of elements of $F_{n}$ is diskbusting and an easy method for generating diskbusting elements. For example any reduced cyclic word containing $a_{1}^{2} a_{2}^{2} \cdots a_{n}^{2} a_{1}$ as a subword is diskbusting. Notice that this is much simpler than the arguments in [1] for the existence of a diskbusting element. We get the following easy corollary.

Corollary 4. Any diskbusting cyclic word in $F_{n}$ has length at least $2 n$ and there are diskbusting cyclic words of length $2 n$.

In general Theorem 3 combined with Whitehead's Reduction Lemma shows that we can change bases and shorten any finite set $\Omega$ of cyclic words until we have found a free decomposition $F_{n}=F_{i_{1}} * F_{i_{2}} * \cdots * F_{i_{s}} * F_{g}$ and a partition $\Omega=\bigcup_{j=1}^{s} \Omega_{j}$ with $\Omega_{j}$ contained (up to conjugacy) in $F_{i_{j}}$ and $\Omega_{j}$ diskbusting in $F_{i_{j}}$. This unfortunately does not directly give Whitehead's conclusion, namely that $\Omega$ is part of a free basis if and only if the Reduction Lemma reduces $\Gamma_{1}(\Omega)$ to a set of vertex-disjoint edges. If $\Omega_{j}$ is part of a free basis for $F_{i_{j}}$, then the diskbusting $\Omega_{j}$ must be a single element and that component of $\Gamma_{1}(\Omega)$ must be a single edge. However a priori it might be the case that $\Omega_{j}$ is part of a free basis for $F_{n}$, but not for $F_{i_{j}}$. There are presumably many ways to show that this cannot occur. However given the fundamental nature of Whitehead's 1936 papers it seems dangerous to use more recent results to establish it. The following easy argument is an adaptation of Whitehead's argument and gives a more powerful conclusion. The free decomposition found by Whitehead's algorithm is unique, though this is not immediately obvious.

To show this uniqueness we need the following lemma. Suppose $a_{1}, a_{2}, \ldots, a_{n}$ is a free basis for $F_{n}=\pi_{1}(M)$, for some 3-manifold $M$. We will say a collection of disjointly embedded $\pi_{1}$-null surfaces $\Sigma_{1}, \Sigma_{2}, \ldots, \Sigma_{n}$ in $M$ represents $a_{1}, \ldots, a_{n}$ if the word in $F_{n}$ represented by a closed loop $\gamma \in \pi_{1}(M)$ transverse to the $\Sigma_{i}$ can be read off by writing down $a_{i}$ when we cross $\Sigma_{i}$ in the forward direction and $a_{i}^{-1}$ when we cross in the reverse direction. Whitehead gives an inductive proof of this fact; our proof will be more direct.

Lemma 5 (Whitehead). Let $a_{1}, a_{2}, \ldots, a_{n}$ and $b_{1}, b_{2}, \ldots, b_{n}$ be free bases of $F_{n}=\pi_{1}(M)$, where $M=\sharp^{n}\left(S^{1} \times S^{2}\right)$. Then we can find a collection of disjointly embedded 2-spheres $\left\{S_{i}^{2}\right\}$ representing $\left\{a_{i}\right\}$ and a collection of disjointly embedded $\pi_{1}$-null surfaces $\Sigma_{i}$, transverse to $\left\{S_{i}^{2}\right\}$, representing $\left\{b_{i}\right\}$ and with the following property.

$(\sharp)$ The closure of any component of $\Sigma_{i}$ cut along the $\left\{S_{i}^{2}\right\}$ meets each $S_{j}^{2}$ either not at all or in a single circle which bounds in $\Sigma_{i}$.

Proof. Choose disjointly embedded 2-spheres $\left\{S_{i}^{2}\right\}$ representing $\left\{a_{i}\right\}$ and disjointly embedded spheres $\left\{\Sigma_{i}\right\}$, transverse to $\left\{S_{i}^{2}\right\}$, representing $\left\{b_{i}\right\}$. We wish 
to alter the surfaces $\left\{\Sigma_{i}\right\}$ to reduce the number of intersection components with $\left\{S_{i}^{2}\right\}$. If property $(\sharp)$ does not hold, then there must be some component of some $\Sigma_{j}$ cut along $\left\{S_{i}^{2}\right\}$ and some 2-sphere $S_{k}^{2}$ which meets it in two circles. Choose a point on each intersection circle and join these points by an $\operatorname{arc} \nu$ in $\Sigma_{j}$ and an arc $\gamma$ in $S_{k}^{2}$. If $\operatorname{int}(\gamma)$ is disjoint from the other surfaces $\left\{\Sigma_{i}\right\}$ then we can surger $\Sigma_{j}$ along $\gamma$ and reduce the number of intersection components.

Choose $\gamma$ to be transverse to the $\left\{\Sigma_{i}\right\}$ and have minimal number of intersections with these surfaces. Suppose $\gamma$ crosses another component of some $\Sigma_{l}$ cut along the $\left\{S_{i}^{2}\right\}$. Since this component is disjoint from $\nu$, it must cross $\gamma$ twice. By the minimality of the number of intersections of $\gamma$ with $\left\{\Sigma_{i}\right\}$ these two intersections must be on different boundary components of $\Sigma_{l} \cap S_{k}^{2}$. Thus passing to this surface $\Sigma_{l}$ instead of $\Sigma_{j}$ we can choose a new curve $\gamma$ with fewer intersections with the $\left\{\Sigma_{i}\right\}$. Therefore the minimal example must have $\operatorname{int}(\gamma)$ disjoint from the $\left\{\Sigma_{i}\right\}$. Thus eventually we must achieve $(\sharp)$.

Proposition 6. Let $F_{n}=A * B=C * D$ be free product decompositions. Then $A \cap C$ is a free factor of $A$, i.e., $A=(A \cap C) * E$ for some $E$.

Proof. Choose a free basis $a_{1}, a_{2}, \ldots, a_{n}$ for $F_{n}$ where $a_{1}, a_{2}, \ldots, a_{k}$ generate $A$ and $a_{k+1}, a_{k+2}, \ldots, a_{n}$ generate $B$. Choose a free basis $b_{1}, b_{2}, \ldots, b_{n}$ for $F_{n}$ where $b_{1}, b_{2}, \ldots, b_{l}$ generate $C$ and $b_{l+1}, b_{l+2}, \ldots, b_{n}$ generate $D$. By Lemma 5 there exist 2 -spheres $\left\{S_{i}^{2}\right\}_{i=1}^{n}$ representing the $a_{i}$ and disjointly embedded $\pi_{1}$-null surfaces $\left\{\Sigma_{i}\right\}_{i=1}^{n}$ representing the $b_{i}$ and satisfying $(\sharp)$. Suppose $\gamma$ is a loop in $M$ representing an element of $A \cap C$. We may assume $\gamma$ is disjoint from $S_{k+1}^{2}, \ldots, S_{n}^{2}$. We wish to show that we may simultaneously choose $\gamma$ to miss $\Sigma_{l+1}, \ldots, \Sigma_{n}$. Suppose not, then since $\gamma$ intersected with the $\left\{\Sigma_{i}\right\}_{i=1}^{n}$ reads off the expression for $\gamma$ in the basis of $b_{i}$ 's there must be two consecutive intersections with some $\Sigma_{j}, l+1 \leq j \leq n$, with cancelling signs. Let $\nu$ be the arc of $\gamma$ joining them. Since $\operatorname{int}(\nu)$ is disjoint from $\left\{\Sigma_{i}\right\}_{i=1}^{n}$ we can homotop $\nu$ (rel endpoints) to an arc $\alpha$ in $\Sigma_{j}$. By property $(\sharp)$, we may choose $\alpha$ so that when read off in the basis $\left\{a_{i}\right\}$ by recording intersections with $\left\{S_{i}^{2}\right\}_{i=1}^{n}$ we get a reduced word. Therefore there cannot be any intersections of $\alpha$ with $S_{k+1}^{2}, \ldots, S_{n}^{2}$. Thus we may homotop $\gamma$ to remove the cancelling intersections with $\Sigma_{j}$ without introducing any intersections with $S_{k+1}^{2}, \ldots, S_{n}^{2}$. Thus iterating this argument we may arrange that $\gamma$ misses $\Sigma_{l+1}, \ldots, \Sigma_{n}$. Hence $A \cap C$ is the image of loops in $M$ missing $S_{k+1}^{2} \cup \cdots \cup S_{n}^{2} \cup \Sigma_{l+1} \cup \cdots \cup \Sigma_{n}$. Surger $M$ and $\Sigma_{l+1}, \ldots, \Sigma_{n}$ along $S_{k+1}^{2}, \ldots, S_{n}^{2}$. The result $M^{\prime}$ has $\pi_{1}\left(M^{\prime}\right)=A$ and we see that $A \cap C$ is the image of loops in $M^{\prime}$ missing the surgered $\Sigma_{l+1} \cup \cdots \cup \Sigma_{n}$. These are a collection of disjointly embedded $\pi_{1}$-null surfaces in $M^{\prime}$, thus $A \cap C$ is a free factor of $A$.

Since the intersection of free factors is again a free factor, it makes sense to talk about the smallest free factor containing (up to conjugacy) a set of cyclic words. Returning to Whitehead's Theorem suppose that Whitehead's Reduction Lemma gave a free decomposition $F_{n}=F_{i_{1}} * F_{i_{2}} * \cdots * F_{i_{s}} * F_{g}$ and a partition $\Omega=\bigcup_{j=1}^{s} \Omega_{j}$ with $\Omega_{j}$ contained (up to conjugacy) in $F_{i_{j}}$ and $\Omega_{j}$ diskbusting 
in $F_{i_{j}}$. Since $\Omega_{j}$ is diskbusting in $F_{i_{j}}$, in fact $F_{i_{j}}$ must the unique smallest free factor containing $\Omega_{j}$. Hence the $\Omega_{j}$ cannot be part of a free basis unless $\Omega_{j}$ is one element and is a generator for $F_{i_{j}}$. Thus we have established Whitehead's result and for $\Omega$ a single element the result below.

Corollary 7 (Whitehead). A finite set of reduced cyclic words $\Omega$ in $F_{n}$ is a subset of a basis if and only if repeated application of Whitehead's Reduction Lemma reduces $\Gamma_{1}(\Omega)$ to a union of (vertex-disjoint) edges and isolated vertices.

Corollary 8. For any element $\omega \in F_{n}$ there is a unique smallest free factor of $F_{n}$ containing $\omega, \omega$ is diskbusting in this free factor and there is an algorithm for finding this free factor.

\section{Ordinary words}

To extend the results above to ordinary words one can proceed as follows. Given a finite set $\Omega=\left\{\omega_{1}, \omega_{2}, \ldots\right\}$ of ordinary words in $F_{n}=\left\langle a_{1}, a_{2}, \ldots, a_{n}\right\rangle$ we can construct analogous graphs. In the handlebody $H$ with cutting 2-disks $D_{1}, D_{2}, \ldots, D_{n}$ fix a base point $\star$ and based curves $\gamma_{i}$ representing $\omega_{i}$. Lift these to the universal cover $\tilde{H}$. For any radius $R$ we may form the graph $\Gamma_{R}^{\text {ord }}(\Omega)$ as follows. Cut along the 2 -disks corresponding to the sphere of radius $R$. Collapse these 2 -disks to vertices and make each copy of $\star$ a vertex. Denote by $\star$ the lift of $\star$ corresponding to the identity element and by $g \star$ the lift corresponding to the group element $g$. Make each segment of each $\gamma_{i}$ an edge. As for the cyclic case the graph $\Gamma_{1}^{\text {ord }}(\Omega)$ was introduced by Whitehead. Whitehead's Reduction Lemma goes through much as before, with one slight change. If the vertex $\star$ is in the component of $\Gamma_{1}^{o r d}(\Omega)-\left\{e_{1}, e_{2}, \ldots, e_{k}\right\}$ containing $x$ then in addition to the change of basis for $F_{n}$ we also replace each $\omega_{i}$ by $x^{\prime} \omega_{i} x$. Then we as above we may always reduce $\Gamma_{1}^{\text {ord }}(\Omega)$ until it is connected and the only possible cut vertex is the base point $\star$. Finally mimicking the proof of Theorem 3 gives the following result.

Theorem 9. Let $\Omega=\left\{\omega_{1}, \omega_{2}, \ldots\right\}$ be a finite set of reduced, ordinary words in $F_{n}$. Then the following are equivalent.

(1) $\Gamma_{1}^{\text {ord }}(\Omega)$ with respect to some basis for $F_{n}$, is connected and has no cut vertex except possibly the vertex $\star$.

(2) $\Gamma_{R}^{o r d}(\Omega)$ is connected for all $R$.

(3) $\Omega$ is geometrically diskbusting.

The case of algebraically diskbusting sets does not fit as well into this graphical presentation but it can still be made to fit.

Definition. Say that one of the graphs $\Gamma_{R}^{o r d}(\Omega)$ is $\star$-split if there is a splitting of the vertices of $\Gamma_{R}^{o r d}(\Omega)-\{\star\}$ into disjoint sets $X$ and $Y$ such that

(1) $X$ and $Y$ are unions of components of $\Gamma_{R}^{o r d}(\Omega)-\{\star\}$, and

(2) If a lift of $\gamma_{i}$ yields one edge in $\Gamma_{R}^{\text {ord }}(\Omega)$ that joins two vertices in $X$ (resp. $Y$ ) then all edges coming from that lift join vertices in $X$ (resp. $Y$ ). 
For $\Gamma_{1}^{\text {ord }}(\Omega),(2)$ may be replaced by the condition that $X$ and $Y$ are closed under taking inverses. Also in this case, we can phrase the definition more geometrically, though less precisely. We say $\Gamma_{1}^{o r d}(\Omega)$ is $\star$-split if there is a partition $\Omega=\Omega_{1} \cup \Omega_{2}$ such that splitting $\Gamma_{1}^{\text {ord }}(\Omega)$ at $\star$ produces a vertex-disjoint union $\Gamma_{1}^{\text {ord }}\left(\Omega_{1}\right) \cup \Gamma_{1}^{\text {ord }}\left(\Omega_{2}\right)$. For general $R$ a $\star$-splitting is a splitting of $\Gamma_{R}^{\text {ord }}(\Omega)$ at $\star$ with each lift of each element of $\Omega$ entirely on one side of the splitting. With this terminology we have the following theorem.

Theorem 10. Let $\Omega=\left\{\omega_{1}, \omega_{2}, \ldots\right\}$ be a finite set of reduced, ordinary words in $F_{n}$. Then the following are equivalent.

(1) $\Gamma_{1}^{\text {ord }}(\Omega)$ with respect to some basis for $F_{n}$, is connected and has no cut vertex except possibly the vertex $\star$ and is not $\star$-split.

(2) $\Gamma_{R}^{o r d}(\Omega)$ is connected and not $\star$-split for all $R$.

(3) $\Omega$ is algebraically diskbusting.

Proof. $(1) \Rightarrow(2)$. As for Theorems 3 and $9, \Gamma_{R}^{o r d}(\Omega)$ is built inductively from $\Gamma_{1}^{\text {ord }}(\Omega)$ (which is connected and not $\star$-split) by replacing vertices by $\Gamma_{1}^{\text {ord }}(\Omega)-$ $\{x\}$ which is connected. This cannot disconnect or introduce a $\star$-splitting.

$(2) \Rightarrow(1)$. Since $\Gamma_{R}^{o r d}(\Omega)$ is connected for all $R$ by Theorem 9 there is some basis in which $\Gamma_{1}^{o r d}(\Omega)$ is connected and has no cut vertex except possibly the vertex $\star$. By hypothesis it is not $\star$-split in that basis.

$(2) \Rightarrow(3)$. Suppose $\Omega$ is not algebraically diskbusting. Then there is a choice of representative based curves $\gamma_{i}$ for $\omega_{i}$ and an essential 2-disk $D \subset H$ such that $D$ meets $\cup \gamma_{i}$ only (possibly) at $\star$. Any homotopic set of curves $\left\{\gamma_{i}^{\prime}\right\}$ may be obtained from $\left\{\gamma_{i}\right\}$ by isotopy and crossing two arcs of curves transversely. We can modify $D$ to keep it disjoint from the $\gamma_{i}$ throughout this homotopy. When two arcs of these curves cross we must add thin $\pi_{1}$-null tubes to any sheet of $D$ that lies between them. Thus we see that any other set of representatives misses a $\pi_{1}$-null surface $F$ homologous to $D$. Thus in any basis there is such an $F$ and $F$ lifts to a compact surface in $\tilde{H}$ which realizes a $\star$-splitting.

$(3) \Rightarrow(2)$. If $\Gamma_{R}^{o r d}(\Omega)$ is disconnected in some basis, then by Theorem $9, \Omega$ is not geometrically diskbusting, hence not algebraically. If $\Gamma_{R}^{o r d}(\Omega)$ is $\star$-split, then there is a 2-disk $D \subset \tilde{H}$ which meets the lifts of the $\gamma_{i}$ only at $\star$. Thus $D$ projects down to an immersed 2-disk $D^{\prime} \subset H$ which meets $\cup \gamma_{i}$ only at $\star$. Thus $\Omega$ is not algebraically diskbusting.

Note that from the proof of Theorem 10, algebraically diskbusting is also algorithmically decidable. Reduce $\Gamma_{1}^{o r d}(\Omega)$ until it is 1-connected except for possibly the vertex $\star$. If $\Gamma_{1}^{o r d}(\Omega)$ is not $\star$-split in this basis then $\Omega$ is algebraically diskbusting. If there are $\star$-splittings, then we get associated to them a free decomposition of $F_{n}$ as in the cyclic case. We have a decomposition $F_{n}=$ $F_{i_{1}} * F_{i_{2}} * \cdots * F_{i_{s}} * F_{g}$ and a partition $\Omega=\bigcup_{j=1}^{s} \Omega_{j}$ with $\Omega_{j}$ contained in $F_{i_{j}}$ and $\Omega_{j}$ algebraically diskbusting in $F_{i_{j}}$. This decomposition is unique. As for the cyclic words this gives us the following corollary. 
Corollary 11 (Whitehead). A finite set of reduced ordinary words $\Omega$ in $F_{n}$ is a subset of a basis if and only if repeated application of Whitehead's Reduction Lemma reduces $\Gamma_{1}^{\text {ord }}(\Omega)$ to a union of a star graph at $\star$ and isolated vertices.

\section{Acknowledgement}

The author would like to thank Michael Freedman for his assistance.

\section{References}

1. R.D. Canary, Ends of hyperbolic 3-manifolds, J. Amer. Math. Soc. 6 (1993), 1-35.

2. J. H. C. Whitehead, On certain sets of elements in a free group, Proc. Roy. Soc. London. 41 (1936), 48-56.

Department of Mathematics, Rice University, Houston, TX 77251

E-mail address: stong@math.rice.edu 